\title{
Recycling of the general transcription factors during RNA polymerase II transcription
}

\author{
Leigh Zawel, K. Prasanna Kumar, and Danny Reinberg ${ }^{1}$ \\ Howard Hughes Medical Institute, Department of Biochemistry, Robert Wood Johnson Medical School, University \\ of Medicine and Dentistry of New Jersey, Piscataway, New Jersey 08854-5635 USA
}

\begin{abstract}
We have anaiyzed the fate of the RNA polymerase II (RNAPII) general transcription factors during the transition from initiation to elongation using multiple approaches. We demonstrate that all of the basal factors coexist in mature initiation complexes but that following nucleotide addition, this complex becomes disrupted. During this transition, TFIID remains promoter-bound whereas TFIIB, TFIIE, TFIIF, and TFIIH are released. Upon release, TFIIB reassociates with TFIID, reforming the RNAPII docking site, the DB complex. TFIIE is released before formation of the tenth phosphodiester bond. This precedes TFIIH release, which occurrs after the transcription complex reaches +30 . TFIIF is unique in that it is the only basal factor detected in the RNAPII elongation complex. Following its release from the initiation complex, TFIIF has the ability to reassociate with a stalled RNAPII.
\end{abstract}

[Key Words: Transcription initiation; general transcription factors; RNA polymerase II; transcription elongation]

Received March 22, 1995; revised version accepted May 2, 1995.

The process of transcription initiation is enormously complex, requiring concerted interactions between at least six protein factors and RNA polymerase II (RNAPII). This family of factors is collectively referred to as the general transcription factors (GTFs) and includes TFIID, TFIIA, TFIIB, TFIIF, TFIIE, and TFIIH $(\mathrm{Za}$ wel and Reinberg 1993).

Prior to formation of the first phosphodiester bond in the nascent transcript, TFIID, TFIIB and TFIIF assemble at the promoter and facilitate loading of RNAPII on to the DNA. Two GTFs, TFIIE and TFIIH, join the complex after polymerase loading. These GTFs are thought to modulate promoter clearance, the process through which RNAPII exits the promoter region and enters the elongation phase (Goodrich and Tjian 1994; Zawel and Reinberg 1995; P. Kumar and D. Reinberg, in prep.). The notion that initiation complex assembly occurs in a stepwise fashion has been challenged by observations made in the yeast system, in which transcriptionally active RNAPII holoenzyme complexes containing SRB (suppressor of RNA polymerase $\underline{B}$ ) proteins, $\sim 20$ unidentified polypeptides, TFIIF, and other basal factors have been reported (Kim et al. 1994; Koleske and Young 1994). Irrespective of how the initiation complex is assembled, its maturation is followed by strand separation and the formation of an open complex, an event that requires the hydrolysis of ATP (Bunick et al. 1982; Sawadogo and Roeder 1984; Jiang and Gralla 1995). In the presence of

\footnotetext{
${ }^{1}$ Corresponding author.
}

nucleoside triphosphates, RNAPII initiates transcription, clears the promoter, and enters the elongation phase.

Little is known about the fate of the GTFs during the transition from initiation to elongation. Early studies performed with fractionated HeLa cell extracts suggested that the TFIID protein fraction remained associated with the promoter following initiation (Van Dyke et al. 1988, 1989). In contrast, studies employing Drosophila extracts suggested that initiation complexes were disassembled completely following each round of RNAPII transcription (Kadonaga 1990). Luse and co-workers provided insight into this transition by analyzing transcription complexes paused at different distances from the initiation site by DNase I and chemical footprinting (Linn and Luse 1991). These studies suggested that RNAPII transcription complexes were subject to numerous structural alterations during formation of the first 30 phosphodiester bonds.

The advent of highly purified reconstituted transcription systems has allowed a more extensive, factor-byfactor analysis of the initiation/elongation transition. Below, we analyze the behavior of human TFIID, TFIIB, TFIIE, TFIIF, and TFIIH. The importance of this work is underscored by recent studies indicating direct interactions between numerous transcriptional activators and basal factors, including the TATA-binding protein (TBP), TFIIB, and TFIIH (for review, see Zawel and Reinberg 1995). Activation can be defined as an increase in the efficiency of transcription of a particular gene within a 
defined period of time. Bearing this in mind, it is possible that the formation of the first initiation complex is not as important to activation as is increasing the number of RNAPII molecules that are consecutively loaded onto the same promoter. Activators targeting basal factors that are released from the transcription complex could impact dramatically on transcription because they could facilitate resetting of the cycle after the first polymerase clears the promoter.

For numerous reasons, understanding the fate of TFIIE and TFIIH has been a subject of particular interest. TFIIH is the only GTF known to contain enzymatic activities (for review, see Drapkin and Reinberg 1994). The discovery that TFIIH participates in nucleotide excision repair (NER) processes and that two of its subunits, ERCC2 and ERCC3, contain DNA helicase activity (Drapkin et al. 1994; van Vuuren et al. 1994; Wang et al. 1994), has led to speculation that TFIIH might travel with RNAPII during elongation, facilitating DNA unwinding and/or coupling of transcription and NER (Buratowski 1993). Also, TFIIE appears to negatively regulate ERCC3 helicase activity (Drapkin et al. 1994), an activity demonstrated to be essential for RNAPII transcription in yeast (Guzder et al. 1994). Thus, for the ERCC3 helicase to contribute to initiation of transcription, one must invoke either a conformational change that frees ERCC 3 helicase from the influence of TFIIE or the removal of TFIIE from the complex.

In this paper a combination of approaches including transcription from DNA templates attached to solid supports, template competition experiments, and immunoblotting were used to characterize the behavior of each of the GTFs, including TFIIE and TFIIH, following the onset of transcription. Collectively, these studies suggest a fate for each of the GTFs during the transition from initiation to elongation.

\section{Results}

Transcription-competent complexes contain TBP, TFIIB, TFIIE, TFIIF, and TFIIH

The assembly of the RNAPII initiation complex has been studied primarily via gel mobility shift assay (Buratowski et al. 1989; Maldonado et al. 1990; Flores et al. 1992). This work has suggested that all of the basal machinery and RNAPII coexist in the initiation complex prior to the addition of nucleoside triphosphates. Because our investigation of basal factor recycling begins with the mature initiation complex, we wished to verify this conclusion by an independent method. We sought an approach, which unlike gel mobility shift assay, would allow us to assay complexes functionally.

Toward this end, initiation complexes were formed with recombinant TBP, TFIIB, TFIIF, TFIIE, and highly purified RNAPII and TFIIH. The DNA template was linear, contained adenovirus 2 major late promoter (AdMLP) core sequences fused to a U-less cassette of 68 nucleotides (U68), and was immobilized 150 nucleotides upstream of the transcription start site as described previously (Arias et al. 1991). Complexes were rinsed to remove unbound proteins with transcription buffer in the presence or absence of Sarkosyl, a detergent capable of removing nonspecifically bound proteins (Hawley and Roeder 1985). Complexes rinsed under either condition were viable, as specific transcripts were produced upon provision of nucleoside triphosphates (NTPs; Fig. 1, left). Washed initiation complexes were subjected to SDSPAGE and transfered to nitrocellulose membranes. TBP, TFIIB, both the large and small subunits of TFIIE and TFIIF, as well as the 62-, 80- (XP-D/ERCC2), and 90-kD (XP-B/ERCC3) subunits of TFIIH were each detected by Western blot with antibodies raised against each of the above (Figs. 1 and 6A, below; data not shown). The binding of factors was specific, as it was not observed when a DNA fragment lacking both TATA and initiator motifs, that is, a functional core promoter, was used (data not shown). With antibodies against the large subunit of RNAPII, the polymerase was also detected within the complex. Consistent with previous observations indicat ing that the nonphosphorylated form of the enzyme preferentially participated in complex formation $(\mathrm{Lu}$ et al 1991; Chesnut et al. 1992), primarily the nonphosphorylated form was detected in the preinitiation complexdespite the fact that the polymerase preparation used to form the complex contained both the phosphorylated and nonphosphorylated forms (Fig. 1, see Pol II).

Sarkosyl rinsing removed a fraction of each of the polypeptides from the complete complex, an effect that resulted in decreased transcription levels (Fig. 1, cf. lanes 1 and 2 and see Western blots). The large subunit of TFIIF, RAP74, was especially sensitive to this treatment. Following the Sarkosyl wash, less than one-tenth of the RAP74 present in the unwashed complex remained. This did not affect our analyses, as previous studies have established that RAP74 is dispensable for transcription initiation (see Discussion; Chang et al. 1993).

One consideration in interpreting the above result is whether all of the complexes formed are viable. However, the fact that we have been able to detect the release of each factor, with the exception of TFIID, using functional assays (see below), provides more direct evidence that all of the factors are in the preinitiation complex. Thus, having ascertained that all of the basal factors coexisted in the initiation complex prior to the addition of nucleotide triphosphates, we next sought to understand the fate of the basal factors after the onset of transcription. Each factor was expected to have one of three possible fates: It could remain at the promoter region, it could be released from the DNA, or it could travel with RNAPII as part of the elongation complex. To distinguish between the first two possibilities, we performed template competition assays both in solution and on support resins. The last possibility was addressed by developing a method for the isolation and characterization of viable elongation complexes (see below).

TBP/TFIID remains bound, whereas TFIIB is released from the promoter upon initiation of transcription

In template competition assays, DNA templates of different sizes are preincubated separately with a subset of 


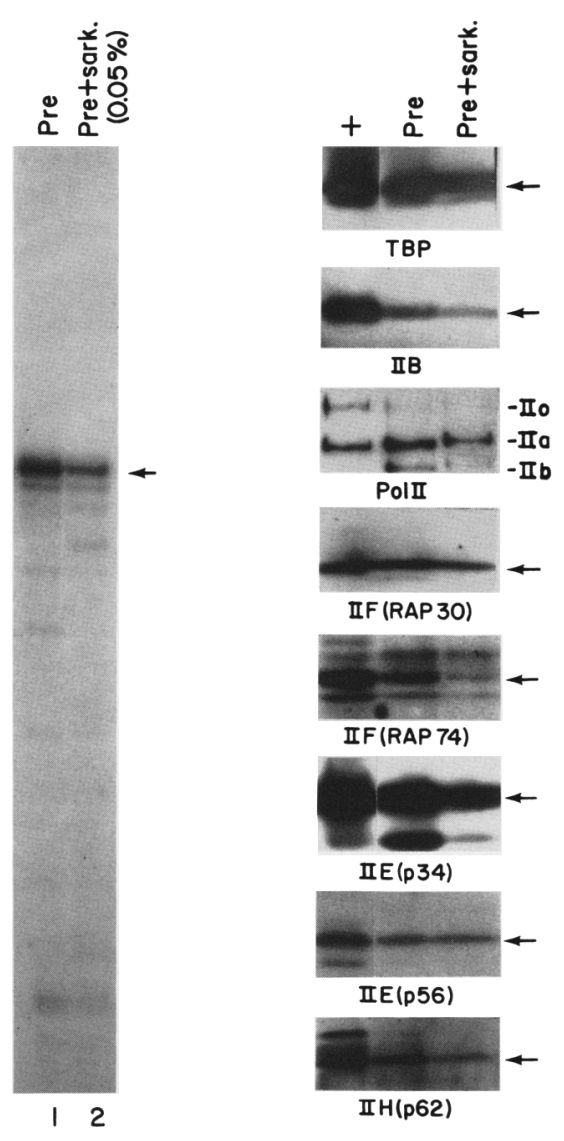

Figure 1. Composition of the preinitiation complex. (Left) Preinitiation complexes were formed on immobilized templates and washed in buffer lacking (Pre, lane 1) or containing $0.05 \%$ Sarkosyl (lane 2), after which NTPS were added to test for transcriptional viability. One-tenth of the reaction was electrophoresed on a urea-polyacrylamide gel. Arrow (right) indicates a 68-nucleotide RNA product. (Right) The remaining nine-tenths of the reaction was subject to SDS-PAGE and transferred to nitrocellulose. Western blots are shown in which complexes were probed for each of the GTFs (bottom of each panel). Antibodies against each of the GTFs have been described previously (Maldonado et al. 1990; Ha et al. 1991; Peterson et al. 1991; Chang et al. 1993; Schaeffer et al. 1993). ( + | Positive control for Western blots. Arrows at right indicate relevant polypeptide for each blot. For RNAPII blot (PolII), a l:1 mixture of the phosphorylated and nonphosphorylated forms were mixed prior to complex formation (Lu et al. 1991). To the right of this blot, IIo, $\mathrm{IIa}$ and IIb correspond to the migration of the phosphorylated, nonphosphorylated, and proteolyzed forms of the largest subunit of RNAPII, respectively. In the TFIIH panel [IIH (p62)], + lane reveals two bands reactive to antibodies against $\mathrm{p} 62$; the upper band represents nonspecific immunoreactive polypeptide. The IIb form of RNAPII detected in initiation complexes, but not in the input sample, is likely the result of proteolysis occurring during complex formation.

initiation factors, one including the factor in question and one without. Because our reconstituted transcription system exhibits a dependence on each of the basal factors (see below), the release or retention of a factor is indicated by the transcription levels of the template lacking the factor in question following template mixing and nucleotide addition. In all of the template competition experiments that were performed in solution, subsaturating levels of the factor being analyzed were used to minimize the possibility that free factor existed in solution.

To determine whether TFIID remained template bound or was recycled, the experiment shown at the bottom of Figure 2A was performed. TBP or TFIID was preincubated on U68 DNA and mixed with a second template, U112 DNA, which had been preincubated with RNAPII and all of the GTFs except TBP/TFIID /see schematic in Fig. 2A, bottom). Nucleotides were added, and transcription from both templates was monitored over time. When either TBP (Fig. 2A, top) or TFIID (middle) was used, $>90 \%$ of the transcription that occurred was directed by U68, even after 90 minutes (lane 4). The absence of transcription from U112 DNA was not the result of inactivation of a GTF, as the addition of TBP after the incubation resulted in transcription (data not shown). Because almost no transcription from U112 was observed, we conclude that TBP/TFIID remains DNA bound following promoter clearance.

TFIIB was analyzed similarly as indicated in Figure $2 B$ (bottom). TFIIB was preincubated on U68 DNA with TBP and TFIIA, a combination of factors that forms a stable preinitiation complex intermediate, the DAB complex (Buratowski et al. 1989; Maldonado et al. 1990). Following template mixing and addition of NTPs and GTFs, TFIIB became available to DNA U112 following a brief lag (cf. lanes 1 and 2). Over the subsequent course of the reaction, each template was transcribed equally. Identical results were obtained when TFIID replaced TBP (data not shown). Similar results were also obtained when TFIIB was preincubated in the context of a complex containing TFIID, TFIIB, RNAPII, and TFIIF (DBPolF), and the acceptor DNA was preincubated with all of the GTFs except TFIIB (data not shown). Thus, in contrast to TFIID, TFIIB appeared to be released some time after template mixing and/or nucleotide addition.

This effect was analyzed further by use of a modified template challenge protocol in which the donor template, the template preincubated with a complete set of factors and RNAPII, was immobilized on a solid support. Immobilizing the donor template allowed us to wash the initiation complexes extensively, virtually ensuring the removal of unbound factors prior to the addition of the acceptor DNA template. Complete initiation complexes were formed on immobilized U112 DNA and washed extensively. In a separate reaction, free U68 DNA was preincubated with a set of factors lacking either TBP or TFIIB. The release of either TBP or TFIIB was measured as a function of the appearance of U68 transcript following mixture of the reactions and addition of nucleotides (see schematic, Fig. 2C). This change in approach did not alter the properties of our system, as initiation complexes formed on the support continued to yield TFIIB transcription activity but not TBP/TFIID (Fig. 2D, cf. U68 transcription in lanes 1 and 2). Importantly, the donor template (immobilized U112 DNA) was transcribed to similar extents in both reactions (lanes 1,2$)$. The re- 


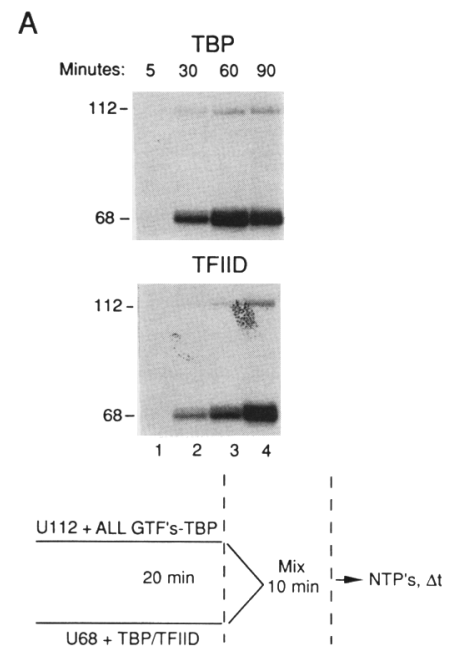

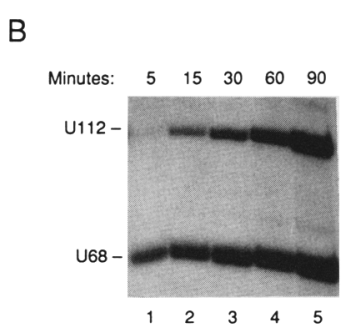

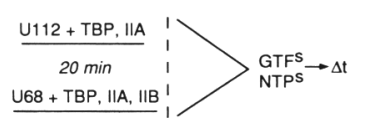

C

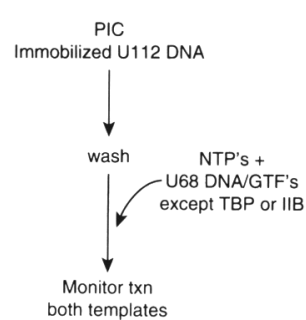

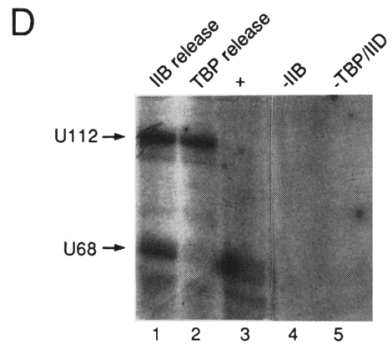

Figure 2. Analysis of the release of TFIID and TFIIB from the promoter by template competition. $(A, B)$ Analysis of TFIID/TBP and TFIIB recycling by template competition assay. The schematic under the autoradiograph indicates which factors were preincubated separately and which factors were added, in addition to NTPs, following template mixing. As indicated in the text, several combinations of factors were tested. RNA synthesis from each template was monitored over time after nucleotide addition. The time, in minutes, at which aliquots were removed from the reaction, is indicated. (A) Analysis of TBP and TFIIID. Limited transcription from U112 DNA appears to be the result of leakiness of the system, as this level was not affected by increasing length of transcription incubation (data not shown). Mobility in 13\% polyacrylamide-urea gels of RNAs derived from U112 and U68 DNAs (I12 and 68). (B) TFIIB analysis. (U112 and U68) mobility of U112 and U68 DNAs, respectively. $(C)$ Diagram of the template competition experiment in which the donor DNA, U112 DNA, was immobilized. (PIC) Preinitiation complex. (D) Lanes 1 and 2 show reaction products from the experiment diagramed in $C$. Lanes 3-5 indicate that transcription of U68 DNA requires both TBP and TFIIB. (U112 and U68) As in $B$.

lease of TFIIB did not occur following the association of RNAPII, TFIIF, TFIIE, and TFIIH with the initiation complex; this event was dependent on the addition of NTPs (data not shown).

\section{Isolation of viable ternary complexes}

Factors TFIIE, TFIIF, and TFIIH each enter the transcription complex with or after RNAPII (for review, see Zawel and Reinberg 1993). Thus, it was important to implement procedures that would allow analysis of factors that might travel with RNAPII during elongation. The strategy illustrated in Figure 3A was developed to isolate and characterize elongation (ternary) complexes. Elongation complexes starved for UTP were formed 68 nucleotides downstream of the transcription start site on immobilized templates (schematic, Fig. 3A). Elongation complexes were recovered by cleavage of the DNA via a restriction site located between the stall site and the promoter region. To facilitate detection of ternary complexes and to assess their viability, radiolabel was incorporated into the nascent RNA. Ternary complexes isolated by this technique were electrophoresed on a native polyacrylamide gel before and after treatment with SDS (Fig. 3B). In the absence of SDS, a major band migrating near the top of the gel was observed (lane 1). This band appeared to consist of nascent RNA in association with elongation complexes, as treatment with SDS resulted in disruption of the complex such that the labeled RNA comigrated with the free RNA at the bottom of the gel (lane 2). Moreover, when ternary complexes were ana- lyzed on denaturing polyacrylamide-urea gels, a predominant band of the correct size, -68 nucleotides, was observed (Fig 3C, lane 1).

Two criteria were used to ensure that complexes isolated in this manner were viable. First, the addition of a nucleotide cocktail including UTP should release the complex from arrest and produce a runoff product of $\sim 84$ nucleotides. As shown in Figure 3C, ternary complexes responded to a UTP-containing nucleotide mixture by elongating the labeled RNA to the end of the linear template (cf. lanes 1 and 2). The second criterion employed was the ability of the complex to carry out a TFIIS-catalyzed backup reaction, which is dependent on ternary complexes (Izban and Luse 1992; Reines 1992). In the absence of NTPs, the addition of TFIIS to stalled ternary complexes results in the activation of a $3^{\prime} \rightarrow 5^{\prime}$ RNAPIIcatalyzed RNA endonuclease (Reines 1992; Gu et al. 1993; Izban and Luse 1993/. Cleavage intermediates of this reaction remain within ternary complexes and can be chased if provided with the necessary nucleotide precursors (Izban and Luse 1992; Reines 1992). As indicated in Figure 3C, lanes 3 and 4, respectively, the ternary complexes isolated were fully competent in executing both the backup and forward reactions in the presence of TFIIS. The decrease in total RNA in lane 4 is likely the result of temary complexes that cleaved the RNA to within 8-9 bases of the RNA 5' end. Such complexes are unstable and, as a result, incapable of resuming transcription (Izban and Luse 1992).

If the ternary complexes isolated were stalled elongation complexes, it is expected that the RNAPII contained 
A

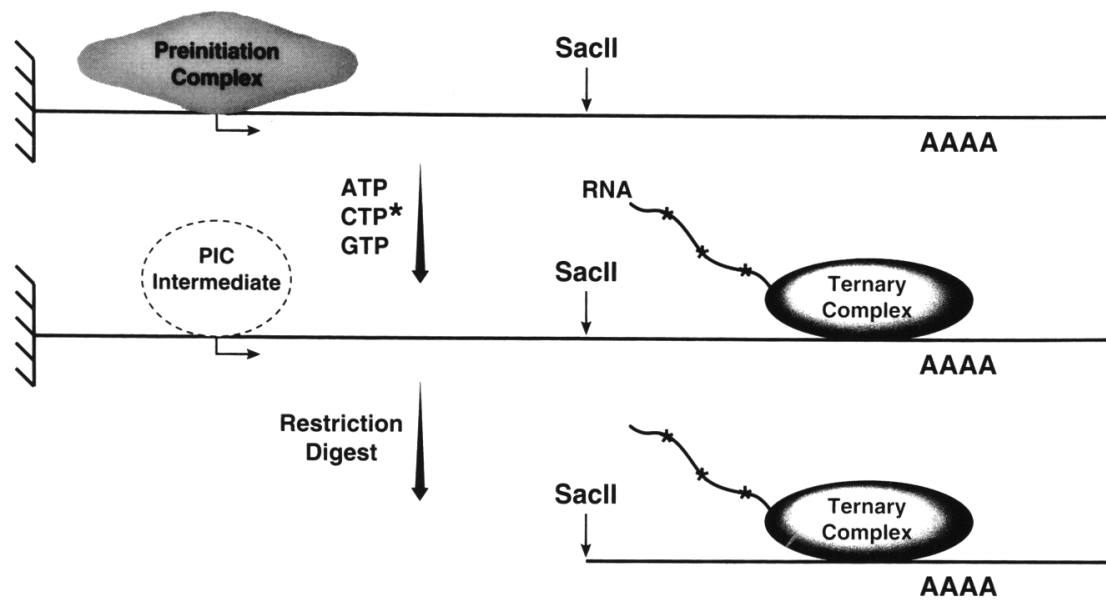

B

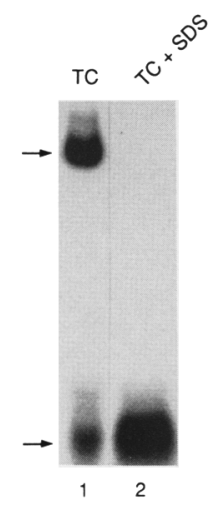

D

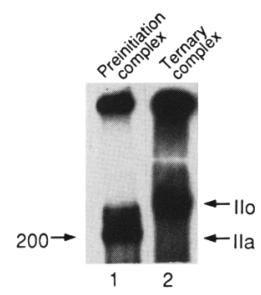

C

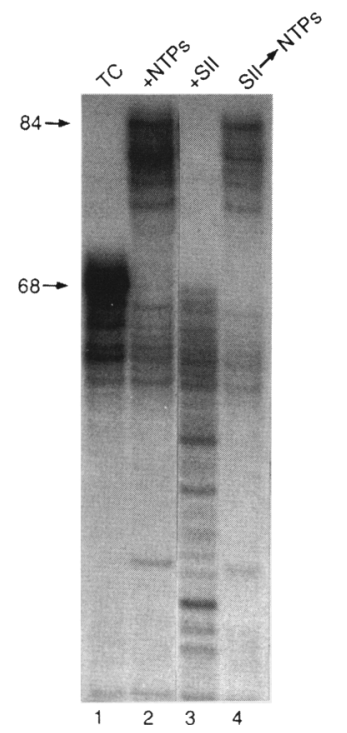

Figure 3. Isolation of viable ternary complexes. $(A)$ Schematic depicting preparation of elongation complexes /see Materials and methods). Initiation complexes were formed on immobilized DNA templates and allowed to clear the promoter by addition of ATP, CTP, and GTP. The asterisk indicates that RNA was radiolabeled by incorporating $\left[\alpha^{-32} \mathrm{P}\right] \mathrm{CTP}$. Because the template contains 4 consecutive A residues at position +68 , elongation complexes will stall in the absence of UTP. Digestion with SacII released ternary complexes, but not promoter-containing DNA, from solid support. $(B)$ Electrophoresis of ternary complexes containing radiolabeled transcript in a $4 \%$ nondenaturing polyacrylamide gel containing $2.5 \%$ glycerol and $0.5 \times$ TBE. (Lane 1) Ternary complex (TC) indicated by arrow; (lane 2) ternary complex treated with $1 \%$ SDS. $(C)$ Ternary complexes were run on denaturing urea-polyacrylamide gels $(15 \%)$. Isolated ternary complexes (lane 1) were incubated with all four ribonucleoside triphosphates (lane 2). In lane 3, complexes were treated for 5 min with TFIIS in the absence of NTPs. Lane 4 was as lane 3 except that NTPs were provided following TFIS treatment. $|68|$ Transcript associated with ternary complex; (84) 84-nucleotide transcript resulting from elongation to the end of the template. $(D)$ Elongation complexes contain the phosphorylated form of RNAPII. RNAPIIA was radiolabeled with $\left[\gamma_{-}{ }^{32} \mathrm{P}\right] \mathrm{ATP}$ and CKII, as described (Chesnut et al. 1992). Complexes were formed with radiolabeled RNAPII and washed as described in Materials and methods. An autoradiograph is shown of a $5 \%$ SDS-polyacrylamide gel in which preinitiation complexes (lane 1) or ternary complexes (lane 2) were electrophoresed. Besides radiolabeled polymerase, no other radiolabel was present in the elongation reaction. (200) Mobility of $200-\mathrm{kD}$ molecular mass marker. (IIo and IIa) Mobilities of phosphorylated and nonphosphorylated forms of RNAPII II subunits. The signal at the top of lanes 1 and 2 results from nonspecific CKII phophorylation.

therein would be highly phosphorylated (Cadena and Dahmus 1987; Payne et al. 1989). To investigate this, RNAPII was radiolabeled by use of casein kinase II (CKII) as described previously (Chesnut et al. 1992). Importantly, CKII phosphorylation does not alter the mobility in SDS gels of the large subunit of RNAPII, as only one phosphate moiety is incorporated at the amino-terminal end of the carboxy-terminal domain (CTD) (Chesnut et al. 1992). CKII-labeled RNAPII was used to generate initiation and ternary complexes as described in Figures 1 and 3, respectively, in the absence of any other radiolabel. Aliquots containing initiation and elongation complexes were subjected to SDS-PAGE. In agreement with previous reports (Cadena and Dahmus 1987; Payne et al. 1989; Lu et al. 1991; Chesnut et al. 1992), only the nonphosphorylated (IIA) form of RNAPII was detected in washed preinitiation complexes (Fig. 3D, lane 1; also see Fig.1), whereas RNAPII derived from isolated ternary complexes was highly phosphorylated (Fig. 3D, lane 2). This observation supported the identity of the isolated complexes as valid elongation complexes.

\section{Analysis of basal factors within elongation complexes}

The above studies demonstrated that TFIID remained promoter bound following the onset of the elongation 
phase, whereas TFIIB was released in a nucleotide-dependent fashion. Thus, in considering the composition of the elongation complex, our initial objective was to probe for TFIIE, TFIIF, and TFIIH.

To analyze for TFIIF, ternary complexes were assayed by Western blot with antibodies against both subunits RAP30 and RAP74. Although RAP30 was detected easily, RAP74 was much less abundant (Fig. 4A). We suspect that this marked difference reflects the removal of the majority of RAP74 in the initiation complex during the Sarkosyl rinsing (Fig. 1). In contrast to TFIIF, neither the 56-, nor the 34-kD subunit of TFIIE was detected in the ternary complex (Fig. 4A).

The above analysis suggested that TFIIF, but not TFIIE, was present in the stalled ternary complex. Thus, we predicted that following initiation, TFIIF would be sequestered in the elongation complex, whereas TFIIE would be released. To test this hypothesis functionally, the template competition experiment diagramed in Figure $4 \mathrm{C}$ was performed. U68 DNA was preincubated with a complete set of GTFs in duplicate reactions. At the same time, two reactions containing U112 DNA were preincubated with GTFs; in these, TFIE or TFIIF was omitted. ATP and CTP were added to all reactions, allowing the polymerase on U68 to elongate up to $10 \mathrm{nu}-$ cleotides before stalling at the first $\mathrm{G}$ residue (Fig. 4B). Addition of ATP and CTP is without effect on U112 DNA, as this complex lacks TFIIE or TFIIF. To determine whether this limited elongation results in the release of TFIIE or TFIIF from U68 DNA, the reactions were mixed to allow for factor exchange between the templates. Transcription was then resumed by the addition of GTP. Heparin was also added to limit transcription to a single round and to ensure that if a factor was released at a point beyond +10 , it would be irrelevant in this assay. As shown in Figure 4D, transcripts originating from U112 DNA appeared in the TFIIE-deficient reaction, but not in the TFIIF-deficient reaction. This was not attributable to TFIIE contamination, as transcription was dependent on TFIIE (lanes 3,4$)$. Nor was this a consequence of free TFIIE in the U68 reaction, as limiting TFIIE levels were employed for this analysis (data not shown). Because TFIIE remained associated with the initiation complex through $0.05 \%$ Sarkosyl washing (Fig. 1), we interpret this as further indication that TFIIE is released following initiation (within the first 10 nucleotides), whereas TFIIF remains associated with a complex that has translocated 10 nucleotides.

\section{TFIIF associates with paused RNAPII complexes}

The notion that TFIF might travel with RNAPII is not without precedent. The interaction between TFIIF and RNAPII is well documented (Flores et al. 1989; Price et al. 1989; McCracken and Greenblatt 1991; Killeen and Greenblatt 1992) as is the ability of TFIIF to stimulate RNAPII elongation rates (Price et al. 1989; Bengal et al. 1991). Despite this evidence, we could not rule out the possibility that TFIIF was released (at a point beyond 10 nucleotides/ and reassociated with stalled RNAPII com-

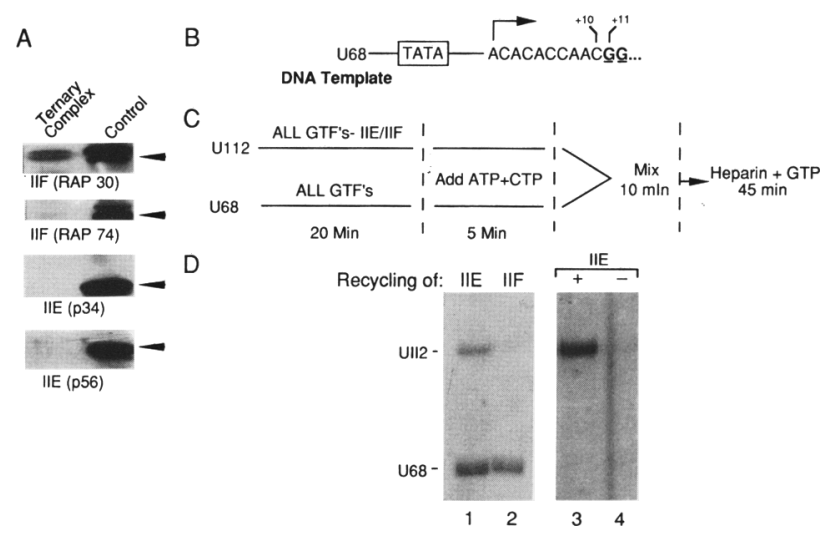

Figure 4. TFIIF, but not TFIIE, is present in ternary complexes. (A) Western blot analysis of the ternary complexes using ECL. Ternary complexes were isolated as in Fig. $3 \mathrm{~A}$, and the proteins were analyzed by Western blot as described in Materials and methods. Visualization of individual polypeptides was accomplished by using antibodies against the factors indicated. Arrowheads indicate positions of the respective polypeptides detected by the antibodies. $(B)$ Schematic of U68 DNA template. The sequence is such that incubation of reaction with ATP and CTP will allow initiation complex to start transcription and translocate to residue +10 , where it will stall in the absence of GTP. (C) Experimental design of template competition experiment shown in D. U68 DNA was preincubated with a complete set of GTFs. In a separate tube, U112 DNA was preincubated with a complete set lacking either TFIIE or TFIIF. During a second preincubation period for $5 \mathrm{~min}$, both reactions were provided with ATP and CTP. The two reactions were mixed for $10 \mathrm{~min}$, after which time GTP and heparin were added. U112 DNA is identical to U68 DNA except the U-less cassette is longer. $|D|$ Autoradiograph of $15 \%$ gel on which reaction products from $C$ were separated. (IIE) Reaction in which U112 DNA did not receive TFIIE; (IIF) reaction in which U112 DNA did not receive TFIIF. (U112 and U68) Locations where transcripts from each template migrate. (Lanes 3,4 ) Transcription reaction is dependent on TFIIE.

plexes. To distinguish between these possibilities, we performed immobilized template competition studies similar to those shown above for TFIIB.

Complete preinitiation complexes were formed on immobilized U68 templates, whereas reactions lacking TFIIF, or RNAPII as a negative control, were preincubated on U112 DNA. After extensively washing the complexes formed on U68 DNA, the U68 and U112 reactions were mixed and a nucleotide cocktail allowing elongation to +68 on the immobilized template (ATP, CTP, and GTP) was provided (Fig. 5A top and bottom left). Because the U112 reaction lacked TFIIF or RNAPII, RNA derived from U112 would only be generated if TFIIF or RNAPII from the U68 template was released and provided to the U112 DNA (Fig. 5B, lanes 2,3). As shown in lanes 4 and 6, U112 RNA was detected when TFIIF, but not RNAPII, was omitted, suggesting release of only the former.

The above experiment indicated that some of the TFIIF that was released assembled into U112 initiation 
A

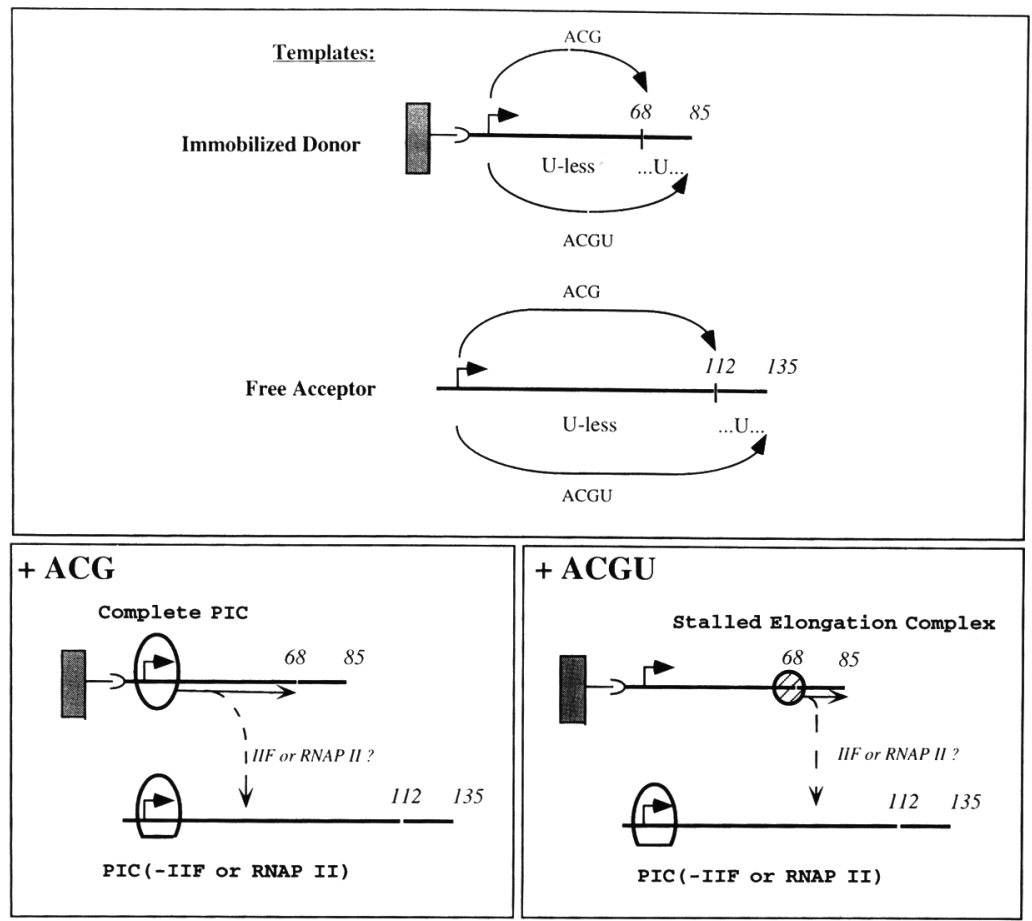

B

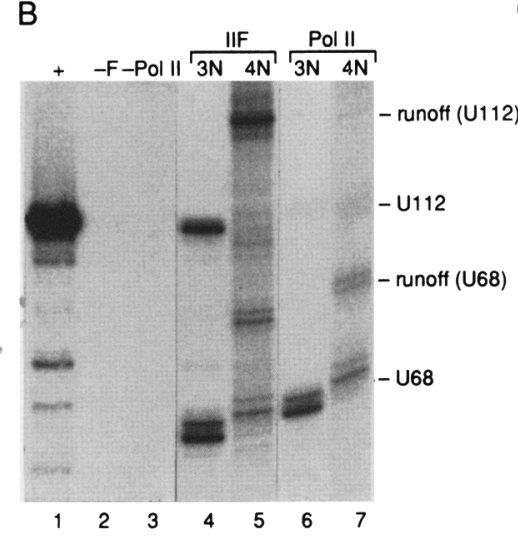

Figure 5. TFIIF is released but reassociates with a stalled RNAPII. (A) Schematic of U-less templates used in analysis (top). U68, which serves as the donor template, was immobilized (indicated by shaded rectangle). U112 template was in solution. Curved arrows indicate transcript length resulting from addition of ATP, CTP, GTP, or all four NTPs. $(68,85,112$, and 135$) \mathrm{Nu}$ cleotide positions along the template. (Bottom left) Experimental design for lanes 4 and 6 of $B$. Complete complexes were formed and rinsed on immobilized U68 template as described in Materials and methods. Complexes lacking either TFIIF or RNAPII were separately preincubated with free U112. Reactions were mixed, and ATP, CTP, and GTP (abbreviated as $3 \mathrm{~N}$ ), were added, allowing the complex on U68 to transit to +68 . The broken line indicates the possible release of TFIIF/ RNAPII from U68. (Bottom right) Experimental design for lanes 5 and 7 in $B$. Elongation complexes stalled at +68 were formed on U68 DNA, whereas initiation complexes lacking either TFIIF or RNAPII were preincubated on U112 DNA as above. The reactions were mixed and all four NTPs $(4 N)$ added. Runoff products for U68 and U112 are 85 and 135 nucleotides, respectively, as indicated at top. The broken line is as above. $(B)$ Reaction products were separated on a $13 \%$ polyacrylamide-urea gel. (Lanes 1-3) Transcription of U112 with all factors $(+)$, in the absence of TFIIF $(-F)$, and in the absence of RNAPII, (-PolII). (Lanes 4-7) Reaction products of experiments diagramed in $A$. Lanes marked IIF, $3 \mathrm{~N}$, and $4 \mathrm{~N}$ indicate that the $\mathrm{U} 112$ reaction lacked TFIIF and contained ATP, CTP, GTP $(3 N)$ or all four nucleoside triphosphates (4N). Lanes marked PolII, 3N, and $4 \mathrm{~N}$ indicate that the $\mathrm{U} 112$ reaction lacked RNAPII, with $3 \mathrm{~N}$ and $4 \mathrm{~N}$, as above. A subpopulation of U68 ternary complexes were not responsive to chasing (lanes 5,7), apparently having become inactivated from the wash treatment. (C) Antibodies against RAP74
coimmunoprecipitate both forms of RNAPII in the presence of TFIIF. The Western blot shown was performed with antibodies against exon 5 of the largest subunit of RNAPII. The RNAPII preparation containing both forms of RNAPII (lane 1) was immunoprecipitated with protein A-immobilized anti-RAP74 antibodies in the presence (lane 3) and absence (lane 2) of TFIIF. (IIo and IIa) Mobility of phosphorylated and nonphosphorylated subunits, respectively.

complexes. To determine whether some of the released TFIIF also reassociated with the stalled elongation complex (as the Western blot analysis indicated), we performed the experiment illustrated in Figure 5A (bottom right). Preinitiation complexes were formed, washed, and allowed to initiate transcription and elongate to +68 . Complexes stalled at +68 were washed again with Sarkosyl to remove factors that had been released during the transition from initiation to elongation. To determine whether TFIIF was contained in the stalled complex, but released upon return to the elongation phase, the complex was allowed to resume elongation by the addition of all four NTPs and mixed with a second reac- tion containing U112 DNA and all of the factors except TFIIF (Fig. 5A, bottom right). As indicated at the top of Figure 5A, addition of all four NTPs allows the formation of runoff products of 85 and 135 nucleotides from the U68 and U112 DNAs, respectively. U112 runoff product was detected when TFIIF release, but not RNAPII release, was analyzed (cf. U112 runoff in lanes 5 and 7). Importantly, the stalled elongation complex on U68 was viable, as addition of all four NTPs chased the RNA from 68 to 85 nucleotides in length (lanes 4,5 and $6,7)$. These data suggest that TFIIF is released during elongation (after +10 ) but has the ability to reassociate with a stalled elongation complex, a result confirmed by 
Western analysis. Moreover, upon resuming elongation, TFIIF is released from the stalled elongation complex.

One prediction from these studies is that TFIIF should be capable of interacting with the nonphosphorylated form of RNAPII, which is present in the initiation complex, as well as the phosphorylated form of RNAPII, present in the elongation complex. This was demonstrated to be the case as measured by coimmunoprecipitation with antibodies raised against the large subunit of TFIIF, RAP74. Both forms of RNAPII were coimmunoprecipitated by anti-RAP74 antibodies in the presence (Fig. 5C, lane 3), but not in the absence (lane 2), of TFIIF.

\section{TFIIH is released during elongation}

Determination of whether TFIIH was part of the elongation complex was one of our primary objectives. TFIIH contains 8-10 subunits /Gerard et al. 1991; Schaeffer et al. 1993), several of which participate in NER (Drapkin et al. 1994; van Vuuren et al. 1994), and is the only GTF known to contain enzymatic activities including a $\mathrm{ki}$ nase that can phosphorylate the CTD of RNAPII and an ATP-dependent DNA helicase activity (for review, see Drapkin and Reinberg 1994).
We probed ternary complexes with antibodies directed against three different TFIIH subunits, p62, ERCC2, and ERCC3 (Fig. 6A; data not shown). Although ERCC2 and ERCC3 were detected in initiation complexes (lane 3), neither was found in isolated elongation complexes (lanes 2). Identical results were obtained for the $62-\mathrm{kD}$ subunit of TFIIH (data not shown).

The above analysis suggested that TFIIH was not part of the elongation complex and, hence, was released. We extended this observation by analyzing for TFIIH release functionally, as was done for TFIIB, TFIIE, and TFIIF. Complete initiation complexes were formed on immobilized U68 DNA and then washed, while TFIIH-deficient reactions were preincubated separately on U112 DNA (schematic, Fig. 6B). The reactions were mixed, nucleotides were provided, and TFIIH release from U68 DNA was monitored as a function of the appearance of U112 RNA, a reaction that is dependent on TFIIH (Fig. 6C, lane 2). Again, RNAPII served as a control. Under these conditions, TFIIH, but not RNAPII, was released (cf. U112 RNA in lanes 3 and 5).

The above study indicated that TFIIH was released prior to the elongation complex reaching +68 . As TFIIH is thought to modulate promoter clearance/Goodrich
A

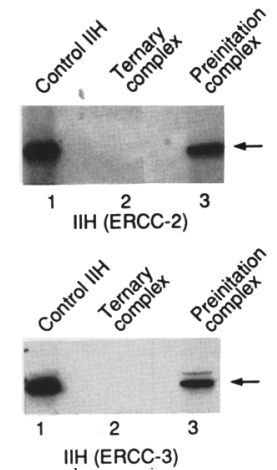

B
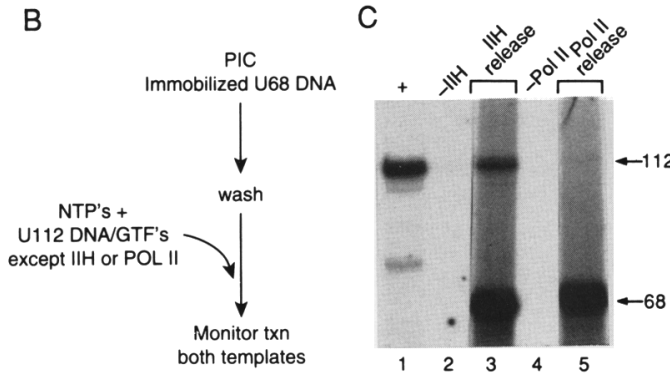

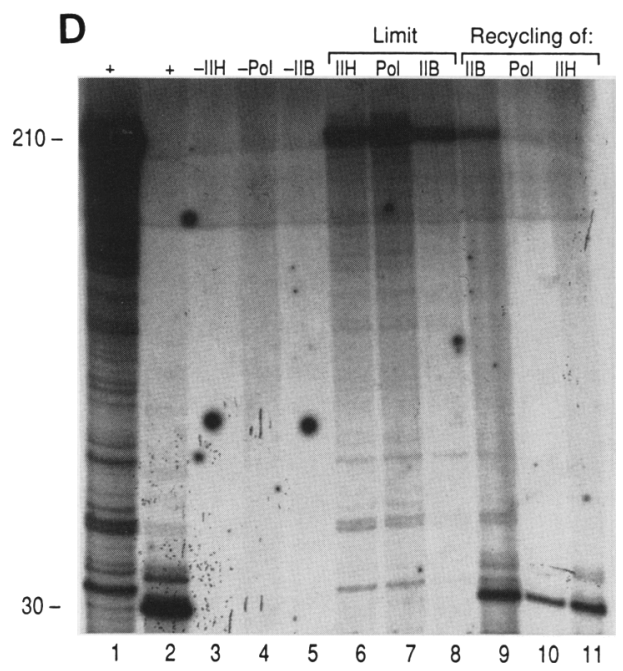

Figure 6. TFIIH is released from the initiation complex. (A) Western blot analysis of the preinitiation complex (lane 3) and the elongation complex (lane 2), formed as described above, with antibodies against the ERCC2 (top) and ERCC3 (bottom) subunits of TFIIH. Complexes were prepared as in Figs. 1 and 3. Control IIH (lane 1) is a positive control for the Western analysis. Arrows point to the mobility of ERCC2 and ERCC3 polypeptides at the top and bottom, respectively. (B) Diagram illustrating the experimental design for the autoradiograph shown in $C$. The complete preinitation complex was formed on immobilized U68 DNA and washed as described in Materials and methods. NTPs and a reaction containing U112 DNA and GTFs but lacking either TFIIH or RNAPII was added simultaneously to washed complexes. Transcription was allowed to proceed for $10 \mathrm{~min}$, after which reaction products were electophoresed on a $13 \%$ polyacrylamide-urea gel. $(C)$ Lanes 1,2 , and 4 show products of transcription reactions containing U1 12 DNA with all GTFs $(+1$, lacking TFIIH $(-$ IIH), and lacking RNAPII - PolII), respectively. Lane 3 (IIH release) shows reaction products from experiment in $B$, where U112 DNA lacked TFIIH. Lane 5 (PolII release) shows products from $B$ in which U112 DNA lacked RNAPII. (112 and 68) Mobility of 112- and 68-nucleotide RNA products. The difference in transcription levels from U68 and U112 likely reflects the time allowed for preinitiation complex formation on U68, but not on U112. $(D)$ Mapping of TFIIH release by template competition in solution. Lanes 1 and 2 are transcription reactions containing all GTFs (+) and either G210 (lane 1) or G30 (lane 2) DNA. Lanes 3-5 reactions contain G210 and lacked factor (indicated at top) Lanes 6-8 contain G210 DNA, and factor (indicated at top) was limited (cf. with lane 1). Lanes 9-11 reactions contain template competition assay, where G30 contained all factors and G210 lacked factor (indicated at top). (30 and 210) Mobility of 30- and 210-nucleotide RNAs. All DNA templates contained Ad MLP fused to the cassette of the size indicated. 
and Tjian 1994; P. Kumar and D. Reinberg, in prep.), it was important to define more precisely when TFIIH was released. To accomplish this, a series of template competition experiments were performed in solution in which the length of the donor template was varied. In all experiments, the acceptor template, the template lacking TFIIH, was linear and produced a transcript of 210 nucleotides (lane 1). Following a brief period of preincubation, the templates were mixed concomitant with the addition of NTPs. Release of TFIIH was measured by the appearance of the 210-nucleotide RNA. TFIIH was not released by complexes allowed to elongate 10,20 , or 30 nucleotides downstream of the initiation site (Fig. 6D, lane 11; data not shown). This was not attributable to inactivation of the acceptor template during the preincubation, as TFIIB (lane 9), but not RNAPII (lane 10), was released under identical conditions. Importantly, transcription was dependent on TFIIH, RNAPII, and TFIIB (lanes 3-5), and subsaturating levels of each of these factors were employed for this analysis (cf. lane 1 with lanes 6-8). Our results were not related to the nature of the transcription cassette used, as similar results were obtained with either U-less or G-less cassettes (data not shown). Thus, TFIIH appears to be the last factor to be released from the transcription complex. This occurs after the complex reaches +30 but before it reaches +68 .

\section{Discussion}

Using a defined reconstituted transcription system and diverse approaches, including transcription of templates attached to solid supports coupled to Western blotting and template competition assay, we have constructed an account of the events preceding and immediately following transcription initiation by RNAPII. Our results shed light on which factors compose a transcription-competent initiation complex, which factors remain bound at the promoter following the release of RNAPII, and which factors are released. A summary of our findings is depicted in the model shown in Figure 7.

We ascertained that five GTFs (TBP, TFIIB, TFIIF, TFIIE, and TFIIH) coexist in the complete initiation complex prior to the addition of NTPs. Although it is possible that some of the complexes analyzed were nonviable, the fact that the release of each factor, with the exception of TFIID, was detected functionally, and, moreover, the fact that we were able to define a point where release occurs, provide further indication that each of the factors is present.

TFIID was found to remain promoter bound through the transcription cycle. These results are in agreement with several previous studies that utilized fractionated HeLa extracts (Van Dyke et al. 1988, 1989; White et al. 1992; Jiang and Gralla 1993) but contradict observations made with Drosophila extracts (Kadonaga 1990). Discrepancies may be attributed to factors such as MOT1 (Auble et al. 1994), which can specifically remove TBP from the DNA. Although TFIID has been implicated as a target for acidic activators (Stringer et al. 1990), several studies have established that binding of TFIID to naked

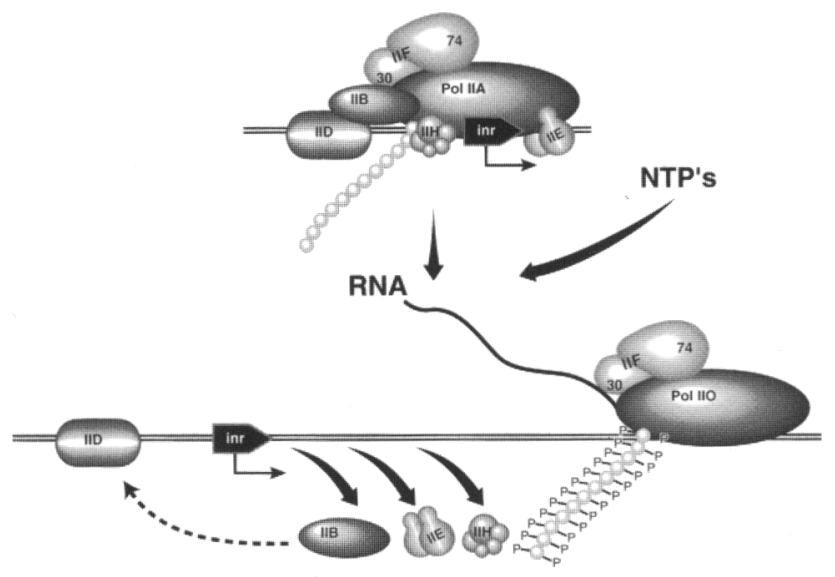

Figure 7. Model depicting the fate of the GTFs during the transition from transcription initiation to elongation. (Top) A preinitiation complex showing all of the GTFs coexisting together with the nonphosphorylated form of RNAPII. Following the onset of elongation, the initiation complex is disrupted. TFIID, or TBP, remains bound at the TATA motif. TFIIB is released but reassociates with TFIID (or TBP). (Bottom) A stalled RNAPII in which the CTD is phosphorylated. TFIIF was released from the initiation complex but reassociates with the stalled polymerase. TFIE and TFIH both recycle with the TFIIE release that occurs first.

DNA is not affected by activators (Choy and Green 1993; Chen et al. 1994). We and others have established that once bound, the interaction of TFIID with the DNA is stable (Van Dyke et al. 1989; White et al. 1992). Thus, activators interacting with TFIID most likely do not affect the binding stability of TFIID. In the case of TFIID, we suspect that the benefit of an activator interaction may be realized only when TFIID binds nucleosomecoated DNA (for review, see Zawel and Reinberg 1995).

TFIIB release was detected immediately upon addition of NTPs, a result consistent with studies from Reines, who demonstrated that TFIIB was not contained in ternary complexes (Reines 1991). The fact that TFIIB is released is somewhat surprising because TFIIB interacts with two components known to interact stably with the promoter, that is, TFIID, via TBP (Ha et al. 1993) and TAF40 (Goodrich et al. 1993), and certain DNA-bound activators (Lin and Green 1991; Choy and Green 1993; Kim and Roeder 1994; Roberts et al. 1995). Rather than maintain TFIIB at the promoter, the activator-TFIIB in teraction apparently stimulates TFIIB assembly (Choy and Green 1993). In agreement with these studies, we have found that the addition of an activator enhances the recruitment of released TFIIB back to the promoter (data not shown).

In an effort to determine whether any basal factors travel with RNAPII during elongation, we developed a procedure for the isolation and analysis of RNAPII elongation complexes. TFIIF was the only initiation factor detected in stalled elongation complexes. TFIIF appears to be released from the initiation complex at some point after formation of the first 10 phosphodiester bonds. Fol- 
lowing its release, TFIIF reassociates with the stalled elongation complex. Upon re-entry of the polymerase into productive elongation, TFIIF is released. Similar findings have been reported in the Drosophila system for the TFIIF homolog, factor 5 (Price et al. 1989). TFIIF is known to interact tightly with RNAPII in solution (Flores et al. 1989; McCracken and Greenblatt 1991). Apparently, this interaction has dual roles. First, TFIIF escorts RNAPII to the assembling initiation complex. The small subunit of TFIIF, RAP30, is independently capable of performing this function (Flores et al. 1991; Killeen et al. 1992). Second, in the event that the polymerase pauses, TFIIF reassociation may serve to facilitate passage through the pause (Price et al. 1989; Bengal et al. 1991). Studies from Burton and co-workers indicated that this property is intrinsic to the large subunit of TFIIF, RAP74, and furthermore, that RAP74 was dispensable for initiation (Chang et al. 1993). This might explain why the observed Sarkosyl-mediated removal of RAP74 from the initiation complex did not appear to compromise transcription in our system.

In agreement with these observations, we found that TFIIF is capable of interacting with both the phosphorylated and nonphosphorylated forms of RNAPII. This is in marked contrast to TBP and TFIIE, which interact only with the nonphosphorylated RNAPII (Usheva et al. 1992; Maxon et al. 1994). Accordingly, we did not detect TFIIE in the elongation complex, where only phosphorylated RNAPII was detected. Rather, TFIIE appeared to be released within the first 10 nucleotides of the nascent RNA. At this time, we cannot determine whether TFIIE is released before, after, or concomitant with TFIIB. Significantly, we have demonstrated that TFIIE release precedes release of TFIIH. This has important mechanistic implications, as TFIIE was found to stimulate TFIIHCTD kinase activity but to negatively regulate TFIIH (ERCC3) helicase activity (Drapkin et al. 1994; Ohkuma and Roeder 1994). Because the helicase associated with ERCC3 was found to be essential for transcription in yeast (Guzder et al. 1994), we suspect that release of TFIIE is a critical checkpoint during initiation.

The investigation of whether TFIIH was in the elongation complex was of particular interest to us. The observation that at least two subunits of TFIIH (ERCC2 and ERCC3) contain DNA helicase activity (Schaeffer et al. 1993; Drapkin et al. 1994) fueled speculation that TFIIH traveled with RNAPII and facilitated DNA unwinding during elongation. Also, recent studies have demonstrated that the TFIIH complex not only participates in transcription initiation but also in DNA excision repair (Drapkin et al. 1994; van Vuuren et al. 1994). Because actively transcribed genes are repaired more efficiently than silent genes, it was thought that perhaps TFIIH was the component that linked these two processes (Buratowski 1993). Contrary to this prediction, neither the p62, p80/ERCC2, or p89/ERCC3 subunit of TFIIH was detected in the ternary complex. Toward reconciling our observations, the following points are offered: With regard to the helicase activity aiding elongation, it is known that RNAPII can transcribe double- stranded DNA extensively in the absence of exogenously added helicase (for review, see Keppola and Kane 1991). In the case of the excision-repair coupling, genetic studies have shown that humans and yeast have specific proteins that couple transcription to repair. CS-A and CS-B (ERCC6) mutants are capable of repairing damaged DNA but are defective in the ability to preferentially repair actively transcribed genes (Troelstra et al. 1992). Although ERCC6 is not a subunit of TFIIH, we have found that it has the ability to interact with TFIIH /C. Selby, R. Drapkin, D. Reinberg and A. Sancar, unpubl.). Thus, when RNAPII stalls at a lesion, ERCC6 may stimulate formation of repair complexes by recruiting TFIIH.

We envision that the release of TFIIH is mechanistically favorable as it ensures that a limited cellular pool of TFIIH will not be sequestered in elongation complexes and is thus available not only to mediate multiple transcription initiation events but also the burden of participating in DNA nucleotide excision repair.

\section{Materials and methods \\ Manipulation of immobilized templates}

The template used for preparing preinitiation and ternary complexes contained the Ad2 MLP fused to a 68-nucleotide U-less cassette. The template was biotinylated by end-filling with biotin-dUTP at the NdeI site 290 bp upstream of the transcription start site. Biotinylated DNA (15 pmoles) was bound to $300 \mu \mathrm{g}$ of streptavidin-coated magnetic beads (Dynabeads M280, Dynal Inc.) in TE buffer. The DNA-bound beads were washed in transcription buffer containing $10 \mathrm{~mm}$ Tris- $\mathrm{HCl}(\mathrm{pH} 7.9), 20 \mathrm{~mm}$ HEPES (pH 7.9), $8 \%$ glycerol, $45 \mathrm{~mm} \mathrm{KCl}, 8 \mathrm{~mm} \mathrm{MgCl}_{2}, 5 \mathrm{~mm}$ $\left(\mathrm{NH}_{4}\right)_{2} \mathrm{SO}_{4}, 2 \% \mathrm{PEG}, 4.5 \mathrm{~mm} \beta$-mercaptoethanol, and $0.05 \mathrm{~mm}$ EDTA. Preinitiation complexes were assembled on immobilized U68 DNA by incubation of the transcription factors TBP, TFIIB, TFIIE, TFIIF, TFIIH, and RNAPII in transcription buffer for $45 \mathrm{~min}$ at $30^{\circ} \mathrm{C}$. For experiments concluding with Western analyses, the amount of protein used for preparing each complex was equivalent to 20 standard transcription assays. For transcription analyses, three standard transcription assays were used. Preinitiation complexes were routinely washed with 5075 volumes of transcription buffer containing $0.05 \%$ Sarkosyl (except where indicated) by repeated concentration and resuspenion with a magnetic stand (Promega). Proteins were eluted with $1 \%$ SDS loading buffer and analyzed by Western blot as described previously (Maldonado et al. 1990). Optimized transcription reactions contained $200 \mathrm{ng}$ of template DNA, $\sim 15 \mathrm{ng}$ of TBP (Maldonado et al. 1990), $5 \mathrm{ng}$ of TFIIB (Ha et al. 1991), 15 ng of TFIIF (Chang et al. 1993), and 8 ng of TFIIE (Peterson et al. 1991), each produced in Escherichia coli and purified as described. Reactions also contained $60 \mathrm{ng}$ of RNAPII (DEAE-5PW step; Lu et al. 1991), $50 \mathrm{ng}$ of TFIIH (Drapkin et al. 1994), and $0.2 \mu \mathrm{g}$ of TFII) (Cortes et al. 1992), each purified from HeLa cells as described previously. TFIIA and native TFIID, when used, were prepared as described (Cortes et al. 1992).

For ternary complex isolation, beads carrying preinitiation complexes were resuspended in transcription buffer containing $1 \mathrm{mM}$ each ATP, CTP, and GTP. After $20 \mathrm{~min}$ of incubation at $30^{\circ} \mathrm{C}$, beads were washed in transcription buffer by repeated concentration and resuspension. Washed beads were resuspended in $150 \mu \mathrm{l}$ of transcription buffer and digested with 120 units of $\mathrm{SaCII}$ at $30^{\circ} \mathrm{C}$ for $1 \mathrm{hr}$. The digest containing the cleaved ternary complex was precipitated with $8 \%$ trichloroacetic acid 
(TCA) on ice, washed with chilled acetone, dissolved in SDSloading buffer, electrophoresed on a $5 \%-17 \%$ gradient polyacrylamide gel, and analyzed by western blot.

\section{In vitro transcription and template competition assay}

DNA templates contained the Ad MLP directing transcription of U-less (U68, U112) or G-less (G10, G20, or G30) cassettes of varying lengths. DNAs were preincubated with a subset of GTFs that differ by one or more factors. Following preincubation, templates were mixed, and nucleotides, together with any required remaining GTF components, were added. Aliquots of reactions were removed at indicated time points. In other respects, transcription reactions were performed as described previously (Lu et al. 1992). Single-round transcription conditions were established by addition of heparin $(10 \mu \mathrm{g} / \mathrm{ml})$ to transcription reactions $3 \mathrm{~min}$ after addition of nucleotides.

\section{Acknowledgments}

We thank the members of the laboratory for discussions and ideas while the work was in progress. Special thanks to Drs. Ronny Drapkin, Aziz Sancar, Ramin Shiekhattar, and Lynne Vales for comments on the manuscript, to Edio Maldonado and R. Weinmann for providing antibodies and to Sasha Akoulitchev for providing artwork. We also thank M. Timmers and R. Tjian for communicating results prior to publication. This work was supported by grants from NIH to D.R. and by the Howard Hughes Medical Institute. L.Z. is supported by an NIH training grant (GM08360).

The publication costs of this article were defrayed in part by payment of page charges. This article must therefore be hereby marked "advertisement" in accordance with 18 USC section 1734 solely to indicate this fact.

\section{References}

Arias, J.A., S.R. Peterson, and W.S. Dynan. 1991. Promoter-dependent phosphorylation of RNA polymerase II by a template-bound kinase. I. Biol. Chem. 266: 8055-8061.

Auble, D.T., K.E. Hansen, C. Mueller, W.S. Lane, J. Thorner, and S. Hahn. 1994. Mot1, a global repressor of RNA polymerase II transcription, inhibits TBP binding to DNA by an ATPdependent mechanism. Genes \& Dev. 8: 1920-1934.

Bengal, E., O. Flores, A. Krauskopf, D. Reinberg, and Y. Aloni. 1991. Role of the mammalian transcription factors IIF, IIS, and IIX during elongation by RNA polymerase II. Mol. Cell. Biol. 11: 1195-1206.

Buratowski, S. 1993. DNA repair and transcription: The helicase connection. Science 260: 37-38.

Buratowski, S., S. Hahn, L. Guarente, and P.A. Sharp. 1989. Five intermediate complexes in transcription initiation by RNA polymerase II. Cell 56: 549-561.

Bunick, D., R. Zandomeni, S. Ackerman, and R. Weinmann. 1982. Mechanism of RNA polymerase II specific initiation of transcription: ATP requirement and uncapped run-off transcripts. Cell 29: 877-886.

Cadena, D.L. and M.E. Dahmus. 1987. Messenger RNA synthesis in mammalian cells is catalyzed by the phosphorylated form of RNA polymerase II. I. Biol. Chem. 262: 1246812474.

Chang, C., Kostrub, C.F., and Z.F. Burton. 1993. RAP30/74 (transcription factor IIF) is required for promoter escape by RNA polymerase II. I. Biol. Chem. 268: 20482-20489.

Chen, J., M. Ding, and D.S. Pederson. 1994. Binding of TFIID to the CYCl TATA boxes in yeast occurs independently of upstream activating sequences. Proc. Natl. Acad. Sci. 91: $11909-11913$.

Chestnut, J.D., J.H. Stephens, and M.E. Dahmus. 1992. The interaction of RNA polymerase II with the adenovirus-2 major late promoter is precluded by phosphorylation of the C-terminal domain of subunit IIa. J. Biol. Chem. 267: 10500 10506.

Choy, B. and M.R. Green 1993. Eukaryotic activators function during multiple steps of preinitiation complex assembly. Nature 366: 531-536.

Cortes, P., O. Flores, and D. Reinberg. 1992. Factors involved in specific transcription by mammalian RNA polymerase II: Purification and analysis of transcription factor IIA and identification of transcription factor IIJ. Mol. Cell. Biol. 12: 413 421.

Drapkin, R. and D. Reinberg. 1994. The multifunctional TFIIH complex and transcriptional control. Trends Biochem. Sci. 19: 504-508.

Drapkin, R., J.T. Reardon, A. Ansari, J.C. Huang, L. Zawel, K. Ahn, A. Sancar, and D. Reinberg. 1994. Dual role of TFIIH in DNA repair and in transcription by RNA polymerase II. $\mathrm{Na}$ ture 368: 769-772.

Flores, O., E. Maldonado, and D. Reinberg. 1989. Factors involved in specific transcription by mammalian RNA polymerase II: Factors IIE and IIF independently interact with RNA polymerase II. I. Biol. Chem. 264: 8930-8921.

Flores, O., H. Lu, M. Killeen, J. Greenblatt, Z.F. Burton, and D. Reinberg. 1991. The small subunit of transcription factor IIF recruits RNA polymerase II into the preinitiation complex. Proc. Natl. Acad. Sci. 88: 9999-10003.

Flores, O., H. Lu, and D. Reinberg. 1992. Factors involved in specific transcription by mammalian RNA polymerase II: Identification and characterization of factor IIH. I. Biol. Chem. 267: 2786-2793.

Gerard, M., L. Fischer, V. Moncollin, J.M. Chipoulet, P. Chambon, and J.M. Egly. 1991. Purification and interaction properties of the human RNA polymerase B (II) general transcription factor BTF2. J. Biol. Chem. 266: 20940-20945.

Goodrich, J.A. and R. Tiian. 1994. Transcription factors IIE, IIH, and ATP hydrolysis direct promoter clearance by RNA polymerase II. Cell 77: 145-156.

Goodrich, J.A., T. Hoey, C.J. Thut, A. Admon, and R. Tjian. 1993. Drosophila $\mathrm{TAF}_{11} 40$ interacts with both a VP16 activation domain and the basal transcription factor TFIIB. Cell 75: 519-530.

Gu, W., W. Powell, J. Mote Jr., and D. Reines. 1993. Nascent RNA cleavage by arrested RNA polymerase II does not require upstream translocation of the elongation complex on DNA. I. Biol. Chem. 268: 25604-25616.

Guzder, S.N., P. Sung, V. Bailly, L. Prakash, and S. Prakash. 1994. Yeast RAD25 gene encodes a DNA helicase required for DNA repair and RNA polymerase II transcription. $\mathrm{Na}$ ture 369: 578-581.

Ha, I., W.S. Lane, and D. Reinberg. 1991. Cloning of a human gene encoding the general transcription initiation factor IIB. Nature 352: 689-695.

Ha, I., S. Roberts, E. Maldonado, X. Sun, L. Kim, M.R. Green, and D. Reinberg. 1993. Multiple functional domains of human transcription factor IIB: Distinct interactions with two general transcription factors and RNA polymerase. Genes \& Dev. 7: 1021-1032.

Hawley, D.K. and R.G. Roeder. 1985. Separation and partial characterization of three functional steps in transcription initiation by human RNA polymerase II. I. Biol. Chem. 
260: 8163-8172.

Izban, M.G. and D.S. Luse. 1992. The RNA polymerase II ternary complex cleaves the nascent transcript in a $3^{\prime} \rightarrow 5^{\prime}$ direction in the presence of elongation factor SII. Genes \& Dev. 6: 1342-1356.

1993. The increment of SII facilitated transcript cleavage varies dramatically between elongation competent and incompetent RNA polymerase II ternary complexes. I. Biol. Chem. 268: 12874-12885.

Jiang, Y. and J.D. Gralla. 1993. Uncoupling of initiation and reinitiation rates during HeLa RNA polymerase II transcription in vitro. Mol. Cell. Biol. 13: 4572-4577.

1995. Nucleotide requirements for activated RNA polymerase II open complex formation in vitro. I. Biol. Chem. 270: $1277-1281$.

Kadonaga, J.T. 1990. Assembly and disassembly of the Drosophila RNA polymerase II complex during transcription. $I$. Biol. Chem. 260: 2624-2631.

Kerppola, T.M. and C.M. Kane. 1991. RNA polymerase: Regulation of transcript elongation and termination. FASEB $/$. 5: 2833-2842.

Killeen, M.T. and J.F. Greenblatt. 1992. The general transcription factor RAP30 binds to RNA polymerase II and prevents it from binding nonspecifically to DNA. Mol. Cell. Biol. 12: $30-37$.

Killeen, M., B. Coulombe, and J. Greenblatt. 1992. Recombinant TBP, transcription factor IIB and RAP30 are sufficient for promoter recognition by mammalian RNA polymerase II. $I$. Biol. Chem. 267: 9463-9466.

Kim, T.K. and R.G. Roeder. 1994. Proline-rich activator CTF1 targets the TFIIB assembly step during transcriptional activation. Proc. Natl. Acad. Sci. 91: 4170-4174.

Kim, Y.-J., S. Bjorklund, Y. Li, M.H. Sayre, and R.D. Kornberg. 1994. A multiprotein mediator of transcriptional activation and its interaction with the C-terminal repeat domain of RNA polymerase II. Cell 77: 599-608.

Koleske, A.J. and R.A. Young. 1994. An RNA polymerase II holoenzyme responsive to activators. Nature 368: 466-469.

Lin, Y. and M.R. Green. 1991. Mechanism of action of an acidic transcriptional activator in vitro. Cell 64: 971-981.

Linn, S.C. and D.S. Luse. 1991. RNA polymerase II elongation complexes paused after the synthesis of 15- or 35-base transcripts have different structures. Mol. Cell. Biol. 11: 15081522.

Lu, H., O. Flores, R. Weinmann, and D. Reinberg. 1991. The non-phosphorylated form of RNA polymerase II preferentially associates with the preinitiation complex. Proc. Natl. Acad. Sci. 88: 10004-10008.

Lu, H., L. Zawel, L. Fisher, J.M. Egly, and D. Reinberg. 1992. Human general transcription factor IIH phosphorylates the C-terminal domain of RNA polymerase II. Nature 358: 641645.

Maldonado, E., I. Ha, P. Cortes, L. Weis, and D. Reinberg. 1990. Factors involved in specific transcription by mammalian RNA polymerase II: Role of transcription factors IIA, IID and IIB during formation of a transcription competent complex. Mol. Cell. Biol. 10: 6335-6347.

Maxon, M., J. Goodrich, and R. Tjian. 1994. Transcription factor IIE binds preferentially to RNA polymerase IIa and recruits TFIIH: A model for promoter clearance. Genes \& Dev. 8: $515-524$.

McCracken, S. and J. Greenblatt. 1991. Related RNA polymerase-binding regions in human RAP30/74 and Escherichia coli $\sigma^{70}$. Science 253: 900-902.

Ohkuma, Y. and R.G. Roeder. 1994. Regulation of TFIIH ATPase and kinase activities by TFIIE during active initia- tion complex formation. Nature 368: 160-163.

Payne, J.M., P.J. Laybourn, and M.E. Dahmus. 1989. The transition of RNA polymerase II from initiation to elongation is associated with phosphorylation of the carboxyl-terminal domain of subunit IIa. J. Biol. Chem. 264: 19621-19629 .

Peterson, M.G., J. Inostroza, M.E. Maxon, O. Flores, A. Admon, D. Reinberg, and R. Tjian. 1991. Structure and functional properties of human general transcription factor IIE. Nature 354: 369-373.

Price, D.H., A.E. Sluder, and A.L. Greenleaf. 1989. Dynamic interaction between a Drosophila transcription factor and RNA polymerase II. Mol. Cell. Biol. 9: 1465-1475.

Reines, D. 1991. RNA polymerase II elongation complex. Elongation complexes purified using an anti-RNA antibody do not contain initiation factor $\alpha$. J. Biol. Chem. 266: 10510 10517.

- 1992. Elongation factor-dependent transcript shortening by template-engaged RNA polymerase II. I. Biol. Chem. 267: 3795-3800.

Roberts, S., B. Choy, S. Walker, Y. Lin, and M.R. Green. 1995. A role for activator-mediated TFIIB recruitment in diverse aspects of transcriptional regulation. Curr. Biol. 5: 508-516.

Sawadogo, M. and R.G. Roeder. 1984. Energy requirement for specific transcription initiation by the human RNA polymerase II system. I. Biol. Chem. 259: 5321-5326.

Schaeffer, L., R. Roy, S. Humbert, V. Moncollin, W. Vermeulen, J. Hoeijmakers, P. Chambon, and J.M. Egly. 1993. DNA repair helicase: a component of BTF2 (TFIIH) basic transcription factor. Science 260: 58-63.

Stringer, K., C.J. Ingles, and J. Greenblatt. 1990. Direct and selective binding of an acidic transcriptional activation domain to the TATA box factor TFIID. Nature 345: 783-786.

Troelstra, C., A.V. Gool, J. Wit, W. Vermeulen, D. Bootsma, and J.H.J. Hoeijmakers, 1992. ERCC6, a member of a subfamily of putative helicases is involved in Cockaynes syndrome and preferential repair of active genes. Cell 71: 939-953.

Usheva, A., E. Maldonado, A. Goldring, H. Lu, C. Houbavi, D. Reinberg, and Y. Aloni. 1992. Specific interaction between the non-phosphorylated form of RNA polymerase II and the TATA binding protein. Cell 69: 871-881.

Van Vuuren, A.J., W. Vermuelen, L. Ma, G. Weeda, E. Appeldoorn, N. Jaspers, A.J. van der Eb, D. Bootsma, J.H.J. Hoefimakers, S. Humbert, L. Schaeffer, and J.M. Egly. 1994. Correction of xeroderma pigmentosum repair defect by basal transcription factor BTF2 (TFIIH). EMBO J. 13: 1645-1653.

Van Dyke, M.W., R.G. Roeder, and M. Sawadogo. 1988. Physical analysis of transcription preinitiation complex assembly on a class II promoter. Science 241: 1335-1338.

Van Dyke, M.W., M. Sawadogo, and R. Roeder. 1989. Stability of transcription complexes on class II genes. Mol. Cell. Biol. 9: 342-344.

Wang, Z., J.Q. Svejstrup, W.J. Feaver, X. Wu, R.D. Kornberg, and E.C. Friedberg. 1994. Transcription factor $b$ (TFIIH) is required during nucleotide excision repair in yeast. Nature 368: 74-76.

White, J., C. Brou, J. Wu, Y. Lutz, V. Moncollin, and P. Chambon. 1992. The acidic transcriptional activator GAL-VP16 acts on preformed template-commited complexes. EMBO J. 11: $2229-2240$

Zawel, L. and D. Reinberg. 1993. Initiation of transcription by RNA polymerase II: A multi-step process. Prog. Nucleic Acid Res. Mol. Biol. 44: 67-108.

1995. Common themes in the assembly of eukaryotic transcription complexes. Annu. Rev. Biochem. 64: 533-561. 


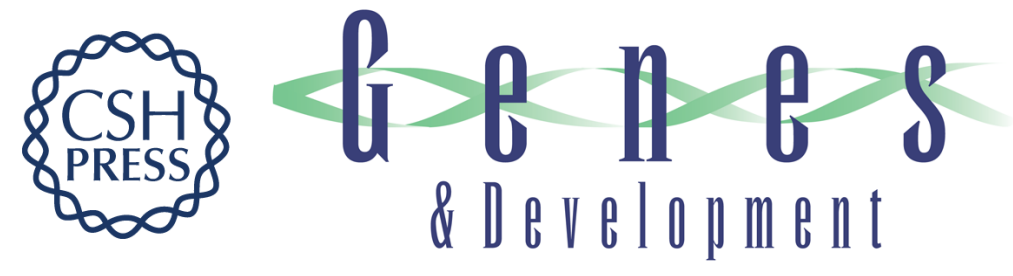

\section{Recycling of the general transcription factors during RNA polymerase II transcription.}

L Zawel, K P Kumar and D Reinberg

Genes Dev. 1995, 9:

Access the most recent version at doi:10.1101/gad.9.12.1479

References This article cites 61 articles, 35 of which can be accessed free at:

http://genesdev.cshlp.org/content/9/12/1479.full.html\#ref-list-1

License

Email Alerting

Service

Receive free email alerts when new articles cite this article - sign up in the box at the top right corner of the article or click here.

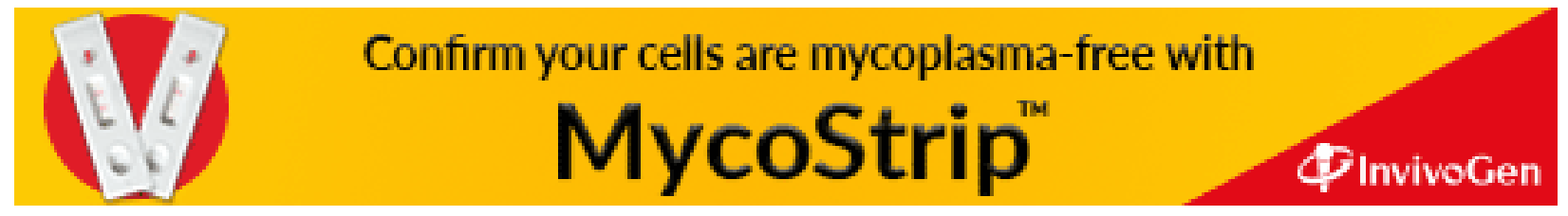

